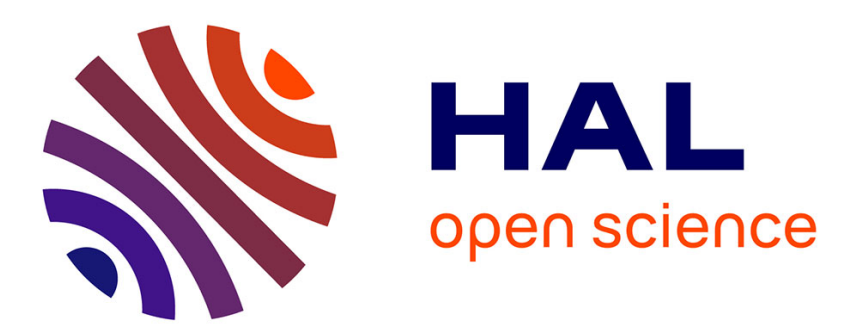

\title{
Usages de l'approche socio-anthropologique du rapport au savoir en sciences de l'éducation et en didactique des sciences : étude comparatiste Pascale Cappiello, Patrice Venturini
}

\section{- To cite this version:}

Pascale Cappiello, Patrice Venturini. Usages de l'approche socio-anthropologique du rapport au savoir en sciences de l'éducation et en didactique des sciences : étude comparatiste. Carrefours de l'éducation, 2011, 31, pp.237-252. halshs-00796065

\section{HAL Id: halshs-00796065 \\ https://shs.hal.science/halshs-00796065}

Submitted on 1 Mar 2014

HAL is a multi-disciplinary open access archive for the deposit and dissemination of scientific research documents, whether they are published or not. The documents may come from teaching and research institutions in France or abroad, or from public or private research centers.
L'archive ouverte pluridisciplinaire HAL, est destinée au dépôt et à la diffusion de documents scientifiques de niveau recherche, publiés ou non, émanant des établissements d'enseignement et de recherche français ou étrangers, des laboratoires publics ou privés. 
Cappiello, P. \& Venturini, P. (2011). Usages de l'approche socio-anthropologique du rapport au savoir en sciences de l'éducation et en didactique des sciences: étude comparatiste. Carrefours de l'éducation, 31, 237-252.

\section{Usages de l'approche socio-anthropologique du rapport au savoir en sciences de l'éducation et en didactique des sciences : étude comparatiste}

Résumé : Cet article présente une comparaison des usages de l'approche socio-anthropologique du rapport au savoir au sein de deux communautés de chercheurs : celle des sciences de l'éducation où elle a vu le jour à l'initiative de l'équipe ESCOL de Charlot, Bautier et Rochex, et celle des didacticiens des sciences qui l'a empruntée. Cette analyse de type épistémologique, précise pour chacun des deux champs, les problématiques traitées, les objets d'études, les méthodologies utilisées et les types de résultats obtenus. Leur comparaison permet de souligner l'intérêt et les limites de l'emprunt réalisé par les didacticiens, ouvre de nouvelles perspectives de recherche et conduit à s'interroger sur les modalités d'emprunt d'un concept à un champ voisin.

Mots-clés : rapport au savoir, rapport aux savoirs scientifiques, épistémologie, didactique des sciences, sciences de l'éducation, perspective comparatiste

Abstract: This article analyses the use of the "relation to knowledge" framework: 1) in the Educational Research community it has been developed on Charlot, Bautier and Rochex's initiative; 2) in the Science Education community which has borrowed it. This epistemological study specifies and compares for each domain, the rationales of the researches, the studied objects, the used methodologies and the types of findings. The paper highlights the interest and the limits of this borrowing, opens new research prospects, and leads us to think about borrowing concepts in a neighbouring field.

Key-words: relation to knowledge; relation to scientific knowledge; epistemology; didactics; Educational Research ; comparative point of view.

L'approche socio-anthropologique du rapport au savoir a été théorisée à la suite de travaux menés en sciences de l'éducation dans les années 80 et 90 puis a été adoptée dans les années 2000 par les didactiques des sciences expérimentales. Nous nous proposons dans cet article d'analyser la manière dont s'est effectué cet emprunt. Nous utiliserons pour cela une approche comparatiste à l'instar d'autres travaux de même type qui ont examiné par exemple, la migration des concepts d'institutionnalisation (Forget, 2008) et de milieu (Weisser, 2009) de la didactique des mathématiques vers la didactique du français, ou encore les usages du concept de transposition didactique dans différentes disciplines (Schubauer-Leoni et Leutenegger, 2005).

Aussi, nous situerons tout d'abord l'approche socio-anthropologique du rapport au savoir dans son contexte d'origine puis dans son champ d'emprunt, ce qui nous permettra de formaliser la question de recherche. Puis nous apporterons des précisons méthodologiques qui permettront de comprendre la nature du travail mené ainsi que la structuration des résultats présentés 
ensuite. Enfin, la conclusion mettra en perspective ces éléments et invitera à une réflexion plus générale sur l'emprunt de concepts à d'autres champs de recherche.

\section{L'approche socio-anthropologique du rapport au savoir, des sciences de l'éducation à la didactique des sciences}

L'approche socio-anthropologique du rapport au savoir $^{1}$ a été initiée dans le champ des sciences de l'éducation par Charlot, Bautier et Rochex (Bautier et Rochex, 1998 ; Charlot, 1997 ; Charlot, 1999 ; Charlot et al., 1992) au sein de l'équipe ESCOL. Elle a été développée à la suite des études menées en sociologie, notamment celles conduites par Bourdieu et Passeron (1970), mettant en relation les difficultés scolaires avec l'existence de handicaps socioculturels. Sans remettre en question ces résultats issus d'une analyse macroscopique de la situation, Charlot et l'équipe ESCOL considèrent l'échec scolaire d'un autre point de vue.

Selon eux, il s'agit surtout "d'une expérience que l'élève traverse, et qu'il interprète » (Charlot, 1997, p. 16), une situation dans laquelle il se trouve au cours de son histoire, dans laquelle le monde, les autres et lui-même sont impliqués. "L'échec scolaire est une situation qui se construit peu à peu, à travers d'autres situations, des pratiques, des rencontres, des événements, des ruptures, des dérives, dans une histoire scolaire inséparable de l'histoire individuelle de l'individu » (Charlot, 1999). L'élève en échec n'est donc pas qu'un élève, identifié par sa position scolaire, c'est «aussi et d'abord, un enfant, un adolescent, c'est-à-dire un sujet, confronté à la nécessité d'apprendre, et à la présence dans le monde de savoirs de divers types »(Charlot, 1997, p. 35). Etudier un sujet dans cette position, en analysant ses propos, sa conduite, sa situation, son histoire..., c'est étudier son rapport au savoir, c'est comprendre quel sens cela a pour lui «d'aller à l'école, de travailler, d'apprendre » (Charlot, 1999, p. 7). Ainsi, la question du rapport au savoir se pose «lorsque l'on constate que certains individus, jeunes ou adultes, ont envie d'apprendre alors que d'autres ne manifestent pas cette envie » (Charlot, 2001, p. 5). Le rapport au savoir " est relation de sens et relation de valeur : l'individu valorise ou dévalorise les savoirs en fonction du sens qu'il leur confère ", et donc se mobilise ou non pour les apprendre (Bautier et Rochex, 1998, p. 34). Du reste, Charlot considère le rapport au savoir avant tout comme un rapport à «l'apprendre » (Charlot, 1997, p. 78).

De leur côté, depuis quelques décennies, les chercheurs en éducation scientifique sont préoccupés à l'égard du peu d'intérêt manifesté selon eux par les élèves envers les disciplines scientifiques. Ainsi de nombreux travaux ont été menés sur les attitudes envers les sciences, principalement à l'étranger (Venturini, 2004a). Le rapport au savoir concernant comme on vient de le dire, la mobilisation à apprendre, il n'est pas surprenant que les didacticiens des sciences aient repris cette approche à leur compte et avec leurs perspectives, tout en la contextualisant aux savoirs scientifiques (Venturini, 2007).

Si les préoccupations au sein des deux communautés de recherche concernent l'envie d'apprendre (ou non) manifestée par les élèves, celles-ci sont inscrites dans une histoire, une culture, et un contexte différent. Ces différences entraînent elles des variations dans l'usage du concept de rapport au savoir et si oui, lesquelles?

Cette question à laquelle nous nous proposons de répondre dans cet article nous conduit aussi à analyser l'intérêt effectif et les limites de cet emprunt pour les didactiques des sciences : en

\footnotetext{
${ }^{1}$ Pour ne pas alourdir la lecture, nous réduirons souvent cette formulation à « rapport au savoir » dans la suite du texte.
} 
quoi et jusqu'où l'approche socio-anthropologique du rapport au savoir a t-elle permis de traiter leurs questionnements et de répondre à leurs attentes, en quoi a-t-elle pu être insuffisante ou encore insuffisamment exploitée ? Les réponses que nous formulerons permettront ainsi de faire un bilan d'étape dans les recherches en didactique des sciences sur ce domaine.

\section{Une analyse de type d'épistémologique}

Notre étude, qui s'intéresse à la manière dont l'utilisation d'un concept permet de produire des savoirs dans deux champs différents, est donc de nature épistémologique ; aussi, elle suppose comme toute analyse épistémologique, une réflexion critique sur les différents travaux des deux communautés, réalisée ici de manière comparative.

Celle-ci a été menée d'une part, à partir des principales publications de l'équipe ESCOL parce que celles-ci sont emblématiques de ce domaine des sciences de l'Education, et d'autre part, à partir des articles utilisant le rapport $\mathrm{au}(\mathrm{x})$ savoir(x) en didactique des sciences. Les références de ces documents qui ont donc constitué notre corpus de données, sont citées tout au long de l'article et précisées dans la bibliographie.

Dans ces différents travaux de recherche, nous avons choisi d'examiner et de caractériser plus particulièrement d'une part les problématiques traitées et les objets d'étude, et d'autre part les méthodologies et les types de résultats obtenus, dans chacun des champs. En effet, cette grille de lecture nous paraît être révélatrice des utilisations d'un concept dans des travaux de recherche et donc susceptible d'éclairer par comparaison, les modalités d'emprunt. Par contre, nous n'avons pas inclus les résultats eux-mêmes dans l'analyse, dans la mesure où ils apparaissent très liés aux contextes de chacune des études.

Examinons donc tout d'abord les problématiques développées dans les deux champs de recherche et leurs objets d'étude.

\section{Comparaison des problématiques et objets d'étude dans les deux champs de recherche}

\subsection{Examen des travaux de l'équipe ESCOL}

Comme nous l'avons précisé plus haut, la notion de rapport au savoir a été introduite par Charlot et l'équipe ESCOL pour mieux comprendre l'échec scolaire. En effet, les résultats obtenus par les sociologues traitant cette question n'expliquent pas ce qu'il se passe en classe pour les élèves en échec, pourquoi ceux-ci ne travaillent pas, n'apprennent pas, pourquoi ils sombrent à l'école dans l'indifférence ou la violence. Ils n'expliquent pas non plus ce que Charlot (2001, p. 7) appelle les "réussites paradoxales », celles d'élèves issus de milieux défavorisés ou, à l'inverse, les échecs paradoxaux d'élèves issus des milieux favorisés. Dans le but de comprendre ces situations, Charlot et l'équipe ESCOL considèrent alors l'élève comme un «sujet humain et singulier inscrit dans un espace social» (Charlot, 1997, p. 91), et le rapport au savoir comme constitué de trois dimensions, épistémique, identitaire, et sociale ${ }^{2}$.

\footnotetext{
${ }^{2}$ La dimension épistémique concerne la question «apprendre, c'est faire quel type d'activité, c'est avoir une activité de quelle nature? (Charlot, 1997, p. 78). La dimension identitaire correspond «à la façon dont le savoir prend sens par rapport à des modèles, à des attentes, à des repères identificatoires, à la vie que l'on veut mener, au métier que l'on veut faire » (Bautier et Rochex, 1998, p. 34). Mais le sujet, dont on étudie le rapport au savoir,
} 
Cette posture particulière, orientée non par la recherche de lacunes ou de handicaps conduisant à l'échec, mais par une «lecture en positif» de l'histoire personnelle du sujet, est caractéristique des travaux d'ESCOL. Chronologiquement, les premiers d'entre eux ont concerné les élèves des collèges et écoles de la banlieue parisienne (Charlot et al., 1992) et ont permis de caractériser :

- les différentes formes de mobilisation vis-à-vis de l'école (par exemple, certains élèves sont mobilisés «sur » l'école, ils donnent du sens au fait d'aller à l'école, mais n'en attribuent pas aux savoirs qu'ils sont censés y apprendre) ;

- les différentes formes de «l'apprendre ${ }^{3} »$ (par exemple, apprendre, ce peut être pour un élève objectiver un savoir, le dénommer, et pour un autre, maîtriser les actions permettant de faire face à une situation particulière sans prendre de recul sur les savoirs en jeu) ;

- les logiques impliquant l'école (par exemple l'école envisagée comme moyen d'accéder à un bon métier ou l'école considérée comme lieu d'acquisition des savoirs).

Ces travaux ont été prolongés d'une part dans les lycées d'enseignement professionnel (Charlot, 1999) et d'autre part dans les lycées d'enseignement général (Bautier et Rochex, 1998).

Dans le premier cas, Charlot a considéré des élèves dominés socialement et scolairement, issus pour la plupart d'une même classe sociale, les milieux populaires. Ces jeunes sont de plus dans une situation particulière : alors que pour les collégiens des milieux populaires, "l'école fait sens en référence au [bon] métier» que l'on obtient «en allant le plus loin possible [dans les études]» (Charlot, 1999, p. 4), les représentations sociales courantes laissent imaginer à ces élèves admis dans un lycée professionnel qu'ils n'ont plus cette perspective. En plus des points examinés dans la recherche menée à l'école et au collège, Charlot a étudié dans ces établissements, le rapport aux savoirs professionnels ainsi que le rapport aux apprentissages effectués dans la cité ou la famille. En effet, "apprendre des mathématiques et apprendre à réparer une moto... mais aussi apprendre tout ce qu'il faut apprendre pour survivre en milieu populaire...non seulement, ce n'est pas apprendre la même chose mais encore ce n'est pas apprendre de la même façon » (Charlot, 2001, p. 7).

Bautier et Rochex se sont intéressés quant à eux aux lycées d'enseignement général. Ils ont cherché à mieux comprendre "ce que sont aujourd'hui la complexité et la diversité de l'expérience lycéenne, particulièrement pour les "publics" qui accèdent dorénavant au second cycle de l'enseignement secondaire alors qu'ils n'y avaient pas ou guère accès il y a 10 ou 15 ans » (1998, p. 64). Pour cela, ils ont étudié "comment les élèves de seconde [les «nouveaux lycéens» mais aussi les autres] vivent et interprètent le lycée, les activités scolaires, ce qu'ils y font et la relation si elle existe entre cette interprétation, la nature du rapport au savoir et aux savoirs (disciplinaires) développé et la construction des savoirs mise en ouvre » (id. p. 45). A cette fin, ils ont enrichi le cadre conceptuel utilisé à l'école et collège d'une "théorie des formes scolaires et de l'activité de l'élève dans leurs rapports aux apprentissages », et «d'une théorie du langage, plus précisément de l'écriture » (id. p. 51). En effet, au lycée existent des formes et des activités scolaires particulières (dissertation, commentaires, etc.), qui mobilisent largement le langage écrit, et qui permettent de construire

a aussi une identité sociale particulière, de par sa position et son histoire sociales. Celle-ci confère une forme particulière aux dimensions épistémique et identitaire (Charlot, 1997, p. 91).

3 Trois processus ont été identifiés par Charlot (1997, p. 80-81), Bautier et Rochex (1998, p. 36) : objectivationdénomination; imbrication du Je dans la situation; distanciation-régulation. 
des connaissances et des modes de pensée, concourant à la construction de soi et autorisant un autre regard sur le monde.

Si au premier abord, les recherches de Charlot et de Bautier et Rochex semblent témoigner d'une divergence dans la problématique et les objets d'étude, une analyse plus attentive nuance largement ce jugement. En effet, les populations concernées présentent des similarités puisque les «nouveaux lycéens » auraient certainement intégré les lycées professionnels il y a une vingtaine d'années. Par ailleurs, dans les deux cas, les chercheurs ont pris en compte des savoirs importants dans chacun des contextes. Ainsi, Charlot s'est intéressé plus particulièrement aux savoirs professionnels qui caractérisent la filière et aux savoirs nécessaires pour survivre dans la cité. Bautier et Rochex ont intégré dans leurs analyses plus spécifiquement les savoirs liés à l'écrit, dont la maîtrise conditionne la réussite au lycée. Enfin, dans les deux cas, il s'agit toujours de comprendre les processus en jeu dans la réussite ou l'échec des élèves.

\subsection{Examen des travaux réalisés en didactique des sciences}

Comme nous l'avons signalé plus haut, les chercheurs en éducation scientifique ont cherché à l'étranger, à comprendre le comportement des élèves en classe, leur engagement à apprendre les sciences. Les cadres qu'ils ont utilisés pour ce faire n'ont pas satisfait totalement leurs attentes, en raison de limitations méthodologiques (travaux sur les attitudes envers les sciences) ou théoriques (travaux sur la motivation) (Venturini 2007, p. 125 et 61). Par ailleurs, dans les travaux auxquels elle a donné lieu, la didactique francophone a considéré uniquement la dimension épistémique des élèves et de ce fait, les résultats qu'elle a obtenus ne permettent pas de comprendre totalement leur comportement dans une classe de sciences, ce modèle étant trop réducteur pour cela. C'est donc parce que dans la théorisation proposée par ESCOL il est question de sens et de valeur du savoir, parce qu'il y est aussi question d'un sujet pluridimensionnel, que les didacticiens des sciences se sont d'abord intéressés aux rapports au savoir des élèves pour tenter de palier les limitations précédentes.

Parce qu'ils ont noté de la part des élèves des mobilisations différentes vis à vis des activités d'apprentissage, les didacticiens ont d'abord supposé que "le rapport au savoir de l'élève intervient comme processus différenciateur du changement conceptuel.» (Chartrain 2002). Aussi, dans un premier temps, ont-ils cherché à établir des relation entre le rapport au savoir des élèves et l'évolution conceptuelle observée à la suite de séances d'enseignement, en particulier sur le volcanisme (Chartrain, 2003) $)^{4}$ ou sur la nutrition des plantes (Catel et al., $2002)^{5}$.

Ces premiers travaux ont alors conduit les chercheurs à s'intéresser aux rapports spécifiques que les élèves entretiennent non plus avec le savoir en général, mais avec les savoirs disciplinaires, centraux dans leurs études. Pour expliciter ces rapports, des recherches ont été menées auprès d'étudiants de licence en physique (Venturini et Albe, 2002) et auprès d'élèves du secondaire, plus particulièrement en $1^{\mathrm{e}} \mathrm{L}$ et en seconde, en physique et en sciences de la vie et de la Terre (SVT) (Cappiello, 2007 ; Rhodes et Venturini, 2006; Venturini, 2005a et 2005b). Par ailleurs, les études de rapports aux savoirs ont permis de confirmer dans le cas de

\footnotetext{
${ }^{4}$ Les travaux de Chartrain ont été menés avec les élèves d'une classe de dernière année d'enseignement primaire (grade 5).

${ }^{5}$ L'étude de Catel, Gallezot et Coquidé a été réalisée d'une part avec des élèves de sixième (grade 6) travaillant sur la production végétale par photosynthèse, et d'autre part avec des élèves de seconde (grade 10) travaillant sur la relation « structure-fonction » du stomate.
} 
la physique l'existence de relations entre rapport aux savoirs disciplinaires et maîtrise conceptuelle (Venturini et Albe, 2002) ${ }^{6}$. Elles ont aussi donné lieu à des études comparatistes entre rapports aux savoirs de différentes disciplines scientifiques (Venturini et Cappiello, 2009) ${ }^{7}$. Enfin, elles ont permis d'identifier des relations entre rapport aux savoirs de la physique et rapport au savoir (Venturini, 2004, 2005a) .

Diminuant encore la granularité de leur objet d'étude, les didacticiens ont aussi mobilisé ce concept pour interpréter les comportements de lycéens à l'égard de savoirs disciplinaires particuliers, concernant la théorie de l'évolution (Chabchoub, 2000 ; Bahloul, 2000 ; Hrairi et Coquidé, 2002) ou la foudre ${ }^{10}$ (Jelman, 2002) dans un contexte culturel fortement marqué ou non par la religion. Ces premiers travaux visaient donc à mieux comprendre le sens donné par les apprenants (élèves ou étudiants) aux savoirs scientifiques pour mieux interpréter leurs comportements en classe de sciences physiques ou de SVT, ou leurs acquisitions conceptuelles.

L'approche ternaire enseignant-apprenant-savoir caractérisant les analyses didactiques, les chercheurs ont aussi envisagé les rapports aux savoirs disciplinaires des professeurs. En effet, ce concept, qui renvoie à la mobilisation des sujets dans des activités mettant en jeu des savoirs peut être envisagé comme un possible « organisateur de la pratique enseignante » comme Bru, Pastré et Vinatier en font l'hypothèse (2007, p. 6). Aussi, d'autres travaux ont mis en relation les rapports aux savoirs disciplinaires des enseignants et certaines de leurs modalités d'intervention dans les classes, en physique (Venturini et al., 2004, 2007) ${ }^{11}$, en mathématiques (Magendie, 2004) et en SVT (Pautal, et al., 2008) ${ }^{12}$. Dans ce dernier cas, il s'agissait de comprendre en quoi les rapports aux savoirs de certains élèves et de leur enseignante pouvaient éclairer une partie de leur action didactique conjointe (Sensevy, 2007)

\subsection{Résultat de la comparaison des problématiques et des objets d'étude}

Les analyses précédentes montrent que la centration des différents travaux est différente. En effet, si ESCOL s'intéresse à un public scolaire en difficulté le plus souvent issu des milieux populaires (écoles et collèges de banlieue, lycées professionnels, nouveaux lycéens), ce sont les savoirs disciplinaires qui restent centraux dans les travaux des didacticiens des sciences. Aussi, on comprend que les chercheurs d'ESCOL aient abordé le rapport au savoir par la dimension sociale même si les derniers travaux de Bautier et Rochex (1998) prenaient davantage en compte certains savoirs disciplinaires du lycée, et que les didacticiens s'en soient approchés d'abord par la dimension épistémique. On peut donc dire à cet égard que ce ne sont pas tout à fait les mêmes sujets qui ont été considérés dans les deux communautés, mais le rapport au savoir a permis aux didacticiens d'élargir le modèle "épistémique » qu'ils utilisaient traditionnellement pour l'élève en prenant en compte une partie de sa pluridimensionnalité, ce qu'ils recherchaient pour mieux interpréter la réalité didactique de la classe. Probablement n'en

\footnotetext{
${ }^{6}$ Cette étude, centrée sur la maîtrise de l'électromagnétisme, a été menée auprès d'étudiants en $3^{\mathrm{e}}$ année d'enseignement supérieur.

${ }^{7}$ L'étude a été menée en sciences physiques et SVT avec des élèves de seconde (grade 10).

${ }^{8}$ Ces travaux ont été réalisés sur des classes de troisième et de seconde (grade 9 et 10)

${ }^{9}$ Ces travaux ont été menés en Tunisie en classe de terminale (grande 12) et en première année universitaire, et pour certains en comparaison avec des élèves français.

${ }^{10}$ L'étude concernant la foudre a été réalisée en Tunisie, en classe de seconde (grade 10).

${ }^{11}$ Ces travaux ont été réalisés en classe de $1^{\mathrm{e}} \mathrm{S}$ (grade 11, classes scientifiques).

${ }^{12}$ Ces deux dernières études ont été réalisées en fin d'école primaire (grade 4 et 5).
} 
ont-ils pas encore totalement exploité les possibilités, notamment la dimension sociale, même si on peut voir dans des travaux récents (Cappiello, 2007) une évolution de ce point de vue ${ }^{13}$. On peut certainement relier ces différences dans la manière de considérer le sujet aux différences dans les problématiques considérées dans les deux communautés, même si à chaque fois il s'agit de comprendre la nature et le degré de mobilisation à apprendre : interpréter des trajectoires scolaires notamment dans le cas d'élèves en échec pour les uns, comprendre le sens donné aux savoirs scientifiques pour les autres. Mais dans ce dernier cas, la problématique a été progressivement affinée : les didacticiens ont en effet aussi cherché à comprendre d'une part si les rapports aux savoirs scientifiques comportaient des spécificités disciplinaires et des généricités interdisciplinaires et d'autre part, comment ceux-ci pouvaient s'articuler au rapport au savoir.

Par ailleurs, ils ont exploré les rapports aux savoirs non seulement d'apprenants (élèves et étudiants) mais aussi d'enseignants du primaire et du secondaire. Dans ce second cas, les sujets sont confrontés à deux types d'activités vis-à-vis des savoirs, non seulement apprendre mais aussi enseigner, et il est difficile d'envisager le « rapport aux savoirs » d'un enseignant sans prendre en compte les deux volets. Si ESCOL a proposé une théorisation rendant compte du rapport à «l'apprendre », il reste encore à proposer pour les usages en didactique un cadre définissant le rapport à "l'enseigner». Il y aurait pour ce faire à analyser en quoi la théorisation existante pourrait être transposée, ou encore en quoi des éléments relevant de ce qu'on appelle avec un certain flou « l'épistémologie professionnelle » (Kagan, 1992, Porlan et al., 1998) pourraient être utiles, au moins pour ceux qui sont directement en lien avec le savoir à enseigner. Le concept emprunté aux sciences de l'éducation montre ici certaines limites par rapport aux besoins exprimés en didactique des sciences, et un travail de formalisation complémentaire semble nécessaire.

Par rapport à la problématique initiale traitée par l'équipe ESCOL, on constate donc la spécificité des problématiques traitées par les didacticiens des sciences (dans lesquelles la nature des savoirs a une grande importance tant au niveau de l'élève que de l'enseignant), et une centration particulière dans la manière de considérer le sujet, actuellement en cours d'évolution. Par ailleurs, l'extension des analyses aux rapports aux savoirs des enseignants s'accompagne d'une évolution dans les pratiques de recherche. En essayant de mettre en relation les rapports aux savoirs des acteurs avec la nature de leur action didactique conjointe (Sensevy et Mercier, 2007), on est passé d'un mode descriptif des rapports au(x) savoir(s) et de leurs liens éventuels avec l'évolution conceptuelle dans un domaine donné à un mode qui se voudrait plus compréhensif des situations didactiques et de leur dynamique.

Après avoir comparé les problématiques et les objets d'étude dans les deux champs, examinons maintenant pour compléter l'analyse, les méthodologies et les types de résultats qu'elles permettent d'obtenir.

\section{Comparaison des méthodologies et des types de résultats associés, dans les deux champs de recherche}

\footnotetext{
${ }^{13}$ Cette évolution correspond d'ailleurs au souhait exprimé par Rochex (2004 p. 99-100) de voir la didactique « se départir de la nä̈veté ou de la cécité sociologique » dont elle fait preuve en ne se souciant pas "des différents contextes sociaux et institutionnels dans lesquels ces activités [de transmission des savoirs et techniques intellectuelles] sont toujours situées ». Ce rapprochement entre les perspectives sociologiques et didactique sur les questions d'éducation est aussi défendu par Joshua et Lahire (1998) et Tupin et Dolz (2008).
} 


\subsection{Examen des travaux de l'équipe ESCOL}

Les chercheurs de l'équipe ESCOL ont mis au point une méthodologie particulière pour mener à bien leurs investigations.

\subsubsection{Recueil de données}

Les problématiques précédentes exigent de recueillir et d'interpréter des données susceptibles de rendre compte du sens que le sujet donne à l'école, aux activités qu'il y mène, à ce qu'il apprend. Il s'agit d'abord d'identifier les phénomènes intervenant dans la mobilisation vis-àvis des apprentissages : actions (travailler à l'école, souvent chez soi), relations (aimer le professeur, faire plaisir à ses parents...), représentations (de l'école, d'une discipline, d'un métier...), mobiles (attentes, demandes, valeurs), psychisme (identifications, projections...), opérations cognitives et langagières (argumenter, catégoriser ...), événements (familiaux, personnels), etc. (Charlot, 1997 p. 34). Il s'agit ensuite de reconstruire les processus résultant de leurs interactions.

Ces informations sont recueillies à l'aide de « bilans de savoir » et d'entretiens individuels.

Dans le bilan de savoir, qui est en fait un bilan de «l'apprendre " (Charlot, 1999, p. 8), les élèves ont à répondre par écrit aux questions suivantes : " j'ai ... ans. J'ai appris des choses, chez moi, dans la cité, à l'école, ailleurs. Qu'est ce qui est important pour moi dans tout ça? Et maintenant qu'est ce que j'en attends? ». Pour l'élève, la question qui se pose alors est «parler de quoi, en parler comment, produire quel type de texte» (Charlot et al., 1992, p. 134). Il est impossible en effet à l'élève de répondre en mentionnant tout ce qu'il a appris et la méthode postule en conséquence que l'élève mentionne les seuls savoirs qui font le plus de sens pour lui. Les bilans de savoir servent à identifier les phénomènes et processus qui se manifestent avec une certaine fréquence et à repérer des élèves présentant des particularités intéressantes à étudier.

Des entretiens semi-directifs sont alors réalisés avec eux pour traiter de leurs histoires singulières. Charlot (1999, p. 12) liste les points abordés avec certains élèves comme ceux des lycées professionnels, parmi lesquels on peut citer l'histoire scolaire de l'élève, le versant familial de cette histoire, l'arrivée au lycée professionnel et ce qu'il en a pensé, ce qu'il en pense maintenant, les relations avec les professeurs et les copains, la définition d'un cours d'un professeur intéressant, etc. L'entretien est aussi un moment de " travail clinique » (mais non thérapeutique) au cours duquel «le sujet puisse, par le biais de notre questionnement de nos reformulations et de leurs effets d'interprétation, être en débat avec lui-même, interroger les rationalisations qu'il opère de sa propre histoire, se poser (voire s'opposer) à lui-même les questions que nous lui posons » (Charlot et al., 1992, p. 241, citant Rochex ${ }^{14}$ 1992).

Quant à eux, Bautier et Rochex (1998, p. 103) ont recueilli en plus, d'autres données auprès des élèves des lycées d'enseignement général : attentes à l'égard de la classe de seconde et de certaines disciplines, attentes à l'égard de la scolarité. A ces écrits déclaratifs s'ajoutent une présentation écrite de la classe de seconde à un élève de troisième, des travaux produits sur consignes scolaires, des notes de cours, ainsi que des observations longitudinales de classes dans plusieurs disciplines.

Ces données ont été analysées et ont permis d'établir un certain nombre de résultats à la forme particulière.

\footnotetext{
${ }^{14}$ Rochex J.-Y., (1992). Entre activité et subjectivité : le sens de l'expérience scolaire. Thèse de doctorat en sciences de l'éducation. Paris : Université Paris 8.
} 


\subsubsection{Modes d'analyse des données et types de résultats}

Le plus souvent Charlot et ESCOL (Charlot et al., 1992, p. 40) utilisent 3 types d'analyses des données :

- « une analyse par thèmes

- une analyse qualitative des pratiques langagières

- une analyse quantitative classique »

D'autres analyses peuvent compléter cet ensemble. Par exemple, Charlot (1999) a pris en compte lors de son étude dans les lycées professionnels, "les éléments relatifs aux lieux auxquels sont référés les apprentissages (école, famille, cité), des éléments relatifs aux personnes permettant l'apprentissage, des éléments liés aux déclarations explicites sur l'école et le savoir quand ils sont présents " (Venturini, 2007, p. 67). Bautier et Rochex ont mis plus fortement l'accent sur les aspects langagiers quand ils se sont intéressés aux nouveaux lycéens.

Les phénomènes mis en évidence sont catégorisés de manière inductive à la suite de l'analyse croisée de plusieurs chercheurs. Il apparaît que certains phénomènes semblent liés, la présence de l'un s'accompagnant généralement de la présence d'un autre. Les chercheurs construisent alors des regroupements basés sur ces cohérences repérées : " on peut ainsi constituer des "constellations d'éléments" et les interpréter comme des ensembles de phénomènes dont l'interaction dynamique constitue un processus » (Charlot et al., 1992, p. 40). Ces typologies de processus en jeu dans "l'apprendre », sont ensuite présentées sous forme d'idéal-types, obtenus "en accentuant unilatéralement un ou plusieurs points de vue et en enchaînant une multitude de phénomènes donnés isolément, diffus et discrets, que l'on trouve tantôt en grand nombre, tantôt en petit nombre et par endroit pas du tout, qu'on ordonne selon les différents points de vue, choisis unilatéralement, pour former un tableau de pensée homogène " (Charlot et al., 1992, p. 41, citant Weber $\left.{ }^{15}, 1965\right)$. Ces idéal-types, « outils conceptuels pour penser les groupes et les individus, sans épuiser leur singularité » (id., p. 41), permettent d'interpréter le type de mobilisation vis-à-vis des apprentissages.

\subsection{Examen des travaux réalisés en didactique des sciences}

Pour mener à bien leurs investigations, les didacticiens des sciences ont adapté la méthodologie mise au point à l'origine par l'équipe ESCOL.

\subsubsection{Recueil de données}

Si sur la forme, les bilans de savoirs et les entretiens avec les élèves ou étudiants ont été conservés, ils ont été contextualisés aux disciplines concernées et enrichis de questions supplémentaires. Par exemple, la formulation usuelle des bilans de savoir est devenue « $j$ ' $a i$ appris des choses en physique à l'école et ailleurs. Qu'est ce qui est important pour moi dans tout ça ? Et maintenant qu'est ce que j'en attends?" (Rhodes et Venturini, 2006; Venturini, 2005a et 2005b). Dans l'entretien individuel destiné à expliciter la dimension sociale du rapport aux savoirs des SVT, les élèves ont dû répondre à "quelle valeur vos parents donnent-ils à l'enseignement des SVT ? Si vous discutez de savoirs des SVT est ce que vos parents sont attentifs? Est-ce que ça les intéresse? Comment réagissent-ils aux résultats scolaires en SVT? » (Cappiello, 2007).

\footnotetext{
${ }^{15}$ Weber M. (1965). Essais sur la théorie de la science. Paris : Plon.
} 
Pour préciser davantage les rapports aux savoirs et ainsi en distinguer plusieurs types, ces modalités de recueil de données ont été complétées dans certaines études par des entretiens sous forme de "focus group» et par des questionnaires ponctuels (Cappiello, 2007). Les premiers étaient destinés à recueillir des données plus fines issues d'échanges et de débats entre pairs (par exemple sur la nature des activités mises en œuvre dans l'apprentissage des SVT), les seconds étaient destinés à préciser certains points ne nécessitant pas un entretien en tête à tête (pratique des médias scientifiques par exemple).

Enfin, les rapports aux savoirs disciplinaires particuliers comme la théorie de l'évolution sont caractérisés à partir des propos des élèves recueillis lors de débats en classe ou de questionnaires les mettant en jeu (par exemple à l'aide des questions «que penses-tu de l'évolution des espèces? Explique ta réponse ») (Hrairi et Coquidé, 2003).

Quant aux rapports aux savoirs des enseignants, ils sont obtenus à partir d'un ou plusieurs entretiens individuels semi-directifs (Magendie, 2004 ; Pautal et al. 2008 ; Venturini et al., 2007). Les professeurs ont ainsi été amenés à évoquer en tant qu'élève (qu'ils ont été) puis en tant qu'enseignant l'intérêt et les attentes vis-à-vis des savoirs de la discipline, les difficultés et/ou les plaisirs liés à leur apprentissage, les activités réalisées pour apprendre ainsi que l'influence sur ces dernières des groupes sociaux auxquels ils appartenaient ou appartiennent maintenant. L'entretien a aussi permis de préciser leur engagement à transmettre ces savoirs disciplinaires à l'école ou à l'extérieur de celle-ci, et ce que l'enseignement de cette discipline représente pour eux. Ces deux derniers éléments marquent pour les savoirs considérés la limite entre le rapport à « l'apprendre » et le rapport à « l'enseigner».

\subsubsection{Modes d'analyse des données et types de résultats}

Ces données, une fois recueillies, ont généralement fait l'objet d'une analyse inductive. Toutefois, dans quelques études, les bilans de savoirs, après segmentation et codage, ont été traités de manière informatique, soit par analyse factorielle des correspondances dans le but de dégager les «configurations» du rapport au savoir (Chartrain 2003), soit par analyse hiérarchique ascendante pour obtenir des classes d'élèves et choisir ceux qui participeront à l'entretien (Cappiello, 2007, Venturini, 2005a et 2005b).

Les résultats sont présentés sous des formes diverses, de manière idéal-typique permettant d'inférer, à l'instar d'ESCOL, une typologie de processus (id.), ou sous la forme d'une typologie basée sur l'attitude comportementale à l'égard de savoirs particuliers (études tunisiennes). Dans le cas des échantillons réduits, ou dans le cas des enseignants, l'idéalisation n'est pas possible et les rapports aux savoirs sont souvent décrits à partir de quelques phénomènes majeurs (Magendie, 2004 ; Pautal et al., 2008 ; Venturini et al., 2007).

\subsection{Résultat de la comparaison des méthodologies et des types de résultats}

Sur le plan méthodologique, les analyses précédentes montrent que, sans remettre en question les fondements de la méthodologie proposée par l'équipe ESCOL, les didacticiens ont apporté quelques innovations sur la formulation des questions, les dispositifs de recueil et de traitement des données. En effet, au-delà de la contextualisation nécessaire aux disciplines scientifiques, les didacticiens ont du faire face à des corpus aux données souvent succinctes et peu discriminantes qui les ont conduits à augmenter le nombre de questions des bilans de savoirs, à tester l'intérêt des focus groups et à utiliser des traitements informatiques pour la classification des données recueillies pour de nombreux élèves.

Par contre, ils ont très peu exploité les possibilités d'analyse langagière, à l'inverse par exemple des travaux de Bautier et Rochex (1998) qui ont utilisé l'écrit scolaire dans certaines disciplines pour préciser le rapport au langage et par là le rapport au savoir. 
En tout état de cause, le rapport $\mathrm{au}(\mathrm{x})$ savoir(s) semble malgré tout délicat à cerner et il est nécessaire de poursuivre la réflexion méthodologique.

\section{Conclusion}

Cette étude a montré que certaines attentes des didacticiens des sciences (comprendre les processus et les phénomènes en jeu dans la mobilisation des élèves à étudier les sciences) peuvent être satisfaites : le rapport au savoir permet de répondre à ce type de préoccupation, même si les recherches sont embryonnaires. Toutefois, on a pu remarquer que certains aspects de la théorisation sont encore peu utilisés dans la formulation des réponses : par exemple, les phénomènes à caractère social sont encore peu intégrés dans les idéalisations des rapports aux savoirs scientifiques. Un certain nombre des potentialités offertes par le concept restent donc encore à exploiter. Par contre, d'autres préoccupations des didacticiens sont plus difficilement prises en compte, en particulier celles qui concernent le rapport au savoir considéré comme un organisateur des pratiques d'enseignement ; en effet, elles nécessitent de définir les rapports $\mathrm{au}(\mathrm{x})$ savoir(s) d'un enseignant ce qui est plus complexe puisqu'ils ne se réduisent pas à un rapport «à l'apprendre ». La théorisation proposée par ESCOL n'envisage pas ce cas qui reste à formaliser. Par ailleurs, si les résultats sont proposés dans chacun des cas sous la forme d'idéal-types, la méthodologie originale proposée par ESCOL a du être modifiée parce qu'elle n'a pas paru totalement adaptée aux caractéristiques des rapports aux savoirs scientifiques dont certains semblent plus difficiles à discriminer que les rapports au savoir. Elle reste cependant à affiner. Ces éléments de conclusion constituent potentiellement autant de perspectives de recherche.

Finalement, de notre point de vue, l'emprunt du concept de rapport au savoir aux sciences de l'éducation a été fécond. Les didacticiens des sciences nous paraissent en avoir tiré profit, et ils ont de plus retourné au champ d'origine des résultats complémentaires (articulation entre rapport au savoir et rapport au savoir disciplinaire) et quelques questions (sur le rapport à «l'enseigner »).

Sur un plan plus large, la comparaison que nous avons réalisée informe sur la dynamique d'appropriation du concept de rapport au savoir par les didactiques des sciences. Nous avons pu voir comment celles-ci ont investi de manière non linéaire les travaux de l'équipe ESCOL et s'en sont nourries, comment elles les ont prolongés en ouvrant de nouveaux chantiers sur les plans théoriques et empiriques. En particulier, on peut voir que l'emprunt de ce cadre théorique à un champ fut-il très voisin, n'est pas neutre. En effet, les didacticiens s'en sont saisi et se le sont approprié à partir et à l'aide de leurs préoccupations, de leurs arrière-plans, de leur culture. Cette composante communautaire pour ne pas dire sociale a modelé leur rapport à cette théorisation, la manière dont ils l'ont appréhendée et utilisée. Il resterait à examiner d'un point de vue épistémologique, si ces processus sont génériques de la manière dont surviennent les emprunts théoriques au sein des Sciences Humaines et Sociales, ou si la nature du cadre théorique emprunté, à l'interface entre la sociologie de l'éducation et les didactiques, ne lui donne pas un caractère plus spécifique.

En tout état de cause, dans les situations de recherche faisant appel à des cadres théoriques extérieurs au champ d'origine, ce ne sont peut-être pas les modalités d'emprunt qui sont les plus importantes, mais plutôt la vigilance épistémologique dont il faut faire preuve pour situer en permanence son activité de recherche par rapport au champ de référence. Pour nous y aider, la démarche comparatiste nous paraît un outil intéressant. 


\section{Références bibliographiques}

Bahloul, M., (2000). Rapports aux savoirs scientifiques et culture d'origine. In A. Chabchoub (Dir.) Rapports au savoir et apprentissage des sciences (pp. 137-148). Tunis: ATRD.

Bautier, E., \& Rochex, J.-Y., (1998). L'expérience scolaire des nouveaux lycéens. Démocratisation ou massification? Paris : Armand Colin.

Bourdieu, P., \& Passeron, J.-C., (1970) La reproduction. Eléments pour une théorie du système d'enseignement. Paris, Editions de Minuit

Bru, M., Pastré, P. \& Vinatier, I. (Dir.) (2007). Les organisateurs de la pratique enseignante, perspective croisées. Recherche et Formation, 56, 5-14.

Cappiello, P. (2007). Rapports entretenus avec les savoirs des sciences de la vie et de la terre par des élèves de seconde. Cas des élèves plus mobilisés sur les apprentissages de la discipline. Mémoire de master 2, non publié, Université de Toulouse.

Catel, L, Coquide, M-L \& Gallezot, M. (2002). Rapport au savoir et apprentissage différencié de savoirs scientifiques de collégiens et de lycéens : quelles questions. Aster, $35 \mathrm{pp}$. $123-148$

Chabchoub, A. (2000). Rapport au(x) savoir(s), didactique des sciences et anthropologie. In A. Chabchoub (dir.) «Rapports aux savoirs et apprentissage des sciences» (pp. 37-46). Tunis : ATRD.

Charlot, B. (1997). Rapport au savoir : Eléments pour une théorie. Paris : Anthropos.

Charlot, B. (1999). Le rapport au savoir en milieu populaire une recherche dans les lycées professionnels de banlieue. Paris : Anthropos.

Charlot, B. (2001). La notion de rapport au savoir : points d'ancrage théoriques et fondements anthropologiques. In B. Charlot, Les jeunes et le savoir, perspectives internationales (pp. 4-24). Paris : Anthropos.

Charlot, B., Bautier, E., \& Rochex, J.-Y., (1992). Ecole et savoir dans les banlieues et ailleurs. Paris : Armand Colin.

Chartrain, J-L. (2003). Rôle du rapport au savoir dans l'évolution différenciée des conceptions scientifiques des élèves. Un exemple du volcanisme au cours moyen 2. Paris: thèse de Doctorat, Université Paris 5.

Hrairi, S. \& Coquidé, M-L. (2003). Attitudes d'élèves tunisiens par rapport à l'évolution biologique. Aster, 35, 149-163.

Jelman Y. (2002). Le rapport aux objets de savoirs comme critère de différenciation entre les apprenants : cas de la foudre. Cahiers pédagogiques : les chroniques du métier. Accessible le 01/08/09 à http://www.cahiers-pedagogiques.com/IMG/pdf/faitsOO6.pdf

Kagan, D. M. (1992). Implications of research on teacher belief. Educational Psychologist, $27(10), 65-70$.

Magendie, L. (2004). Rapport à l'apprendre et pratiques d'enseignement de professeurs d'écoles : étude de cas en mathématiques. Mémoire de master 2, non publié, Université de Toulouse.

Pautal, E., Venturini, P., Dugal, J-P. (2008). Prise en compte du rapport aux savoirs pour mieux comprendre un système didactique. Un exemple en SVT à l'école élémentaire. Didaskalia, 33, 63-88. 
Porlan Ariza, R., Garcia, E., \& Martin del Pozo, E., Les obstacles à la formation professionnelle des professeurs en rapport avec leurs idées sur la science, l'enseignement et l'apprentissage. Aster 26, 207-235.

Rhodes, C., \& Venturini, P. (2006). Analyse du rapport aux savoirs de la physique d'une classe de 1ère L. Communication à la 8e Biennale internationale de l'éducation et de la formation. Lyon 11 au 14 avril 2006.

Rochex, J.-Y. (2004). La notion de rapport au savoir : convergences et débats théoriques. Pratiques psychologiques, 10, 93-106.

Sensevy, G. (2007). Des catégories pour décrire et comprendre l'action didactique. In G. Sensevy \& A. Mercier, (2007) (dir.), Agir ensemble : l'action didactique conjointe du professeur et des élèves (pp. 13-49). Rennes : Presses Universitaires de Rennes.

Sensevy, G., \& A. Mercier, (2007) (dir.). Agir ensemble : l'action didactique conjointe du professeur et des élèves. Rennes : Presses Universitaires de Rennes.

Venturini, P. (2004a). Note de Synthèse : Attitudes des élèves envers les sciences : le point de recherches. Revue Française de Pédagogie, 149, 97-121.

Venturini, P. (2004b). Relations entre les rapports entretenus par des élèves de Troisième avec le savoir scolaire et avec les savoirs de la physique. In actes du $5^{\text {ième }}$ congrès international « Actualité de la recherche en éducation et en formation». CNAM, Paris, 31 août - 4 septembre 2004.

Venturini, P. (2005a). Phénomènes et processus intervenant dans les rapports aux savoirs de la physique : cas d'élèves français en 10ième année de formation. Revue Suisse des Sciences de l'Education, 27(1), 103-121.

Venturini P. (2005b) Rapports idéal-typiques à la physique d'élèves de l'enseignement secondaire. Didaskalia, 26, 9-32

Venturini, P., \& Albe, V. (2002) Interprétation des similitudes et différences dans la maîtrise conceptuelle d'étudiants en électromagnétisme à partir de leur(s) $\operatorname{rapport}(\mathrm{s}) \mathrm{au}(\mathrm{x})$ savoir(s). Aster, 35, 165-188

Venturini, P. (2007). L'envie d'apprendre les sciences. Motivation, attitudes, rapport aux savoirs scientifiques. Paris : Editions Fabert.

Venturini, P., Calmettes, B., Amade-Escot, C., \& Terrisse, A. (2004). Travaux personnels encadrés en 1ère $\mathrm{S}$ à dominante physique : étude de cas et analyse didactique. Aster, 39, $11-37$

Venturini, P., Calmettes, B., Amade-Escot, C., \& Terrisse, A. (2007). Analyse didactique des pratiques d'enseignement de la physique d'une professeure expérimentée. Aster, 45, 211 234.

Venturini, P. \& Cappiello, P. (2009). Comparaison des rapports aux savoirs de la physique et des SVT dans le cas d'élèves impliqués dans l'étude de ces disciplines. Revue Française de Pédagogie, 166 (à paraître) 